\section{LESS INTENSE AND ALCOHOL FREE}

Johnson \& Johnson have launched a less intense, alcohol-free version of Listerine Mouthwash: Listerine Zero. less intense taste, but still contains the classic Listerine four essential oils: menthol, thymol, methyl salicylate and eucalyptol. Listerine Zero kills up to 99\% of plaque bacteria in vitro, more than other alcohol-free, daily use mouthwashes. Listerine Zero also contains 220 ppm (0.05\%) fluoride for effective enamel protection.

According to Johnson \& Johnson, Listerine Zero has been developed to address the individual needs of patients who may, for lifestyle, health or religious reasons, or on grounds of taste, prefer a less intense, alcohol-free mouthwash.
The new product is alcohol-free for a

For dental professionals, there is now a less intense and alcohol-free, yet highly effective daily use mouthwash within the Listerine range to suit the individual needs of some patients and to help them achieve a cleaner, fresher and healthier mouth beyond toothbrushing and interdental cleaning alone.

Reader response number 53

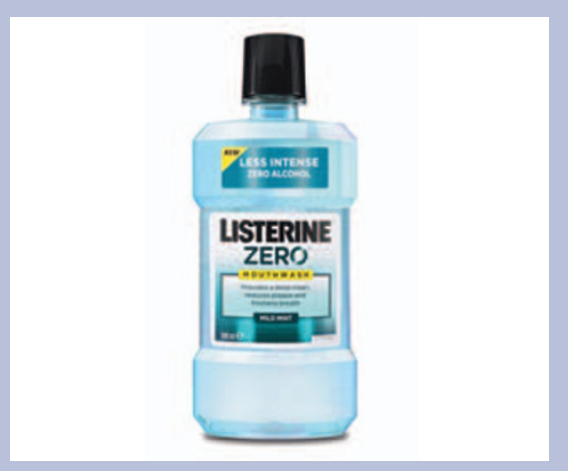

\section{CLEAR ALIGNER INNOVATIONS}

Align Technology, Inc. has launched Invisalign G3 in the UK. Engineered to make Invisalign easier to use with Class II and Class III patients, Invisalign G3 is designed to deliver significantly superior clinical results.

Class II and III treatments make up approximately $40 \%$ of all patients in an orthodontist's practice. In order to treat Class II/III malocclusions, doctors previously had to manually cut the aligners to accommodate the use of elastics, which may have discouraged the use of Invisalign in these cases. Invisalign G3 addresses this barrier with new Precision Cuts, which are doctor-prescribed cuts in the aligners to accommodate the use of orthodontic elastics.

Invisalign G3 also features new Passive Aligners - aligners with no movements which facilitate the use of Class II/III elastics while treatment in the opposite arch finishes. Available for dual arch treatments with Invisalign Full and Invisalign Teen, Passive Aligners allow for smoother management of patient treatments.

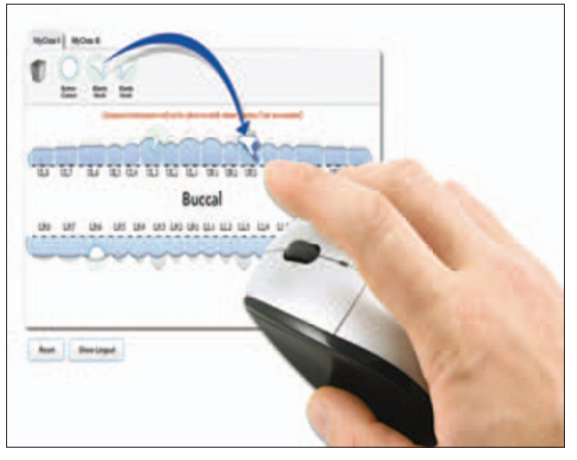

SmartForce features, such as the 0ptimized Attachments, are attachments and aligner features that are engineered to deliver the forces needed to achieve predictable tooth movements. SmartForce features are customised to each tooth based on biomechanical principles, and use advanced virtual modelling in the design of each aligner.

Significant evolution of the ClinCheck software makes it easier and more intuitive to create and optimise Invisalign treatment plans. Using the new dragand-drop interfaces, operators have the flexibility to specify the placement and the type of Precision Cuts and attachments on the aligners.

Reader response number 54

\section{OUTSTANDING CUTTING PERFORMANCE}

For implantologist teams preferring tapered implant-abutment connections, leading implant dentistry provider CAMLOG is expanding its range of products with the launch of its CONELOG Implant System.

CAMLOG implants and CONELOG implants have the same outer geometry (SCREW-LINE implants) and can be inserted using one and the same surgery set. This offers a high degree of flexibility in surgical practice and makes handling of the instruments simpler. The CAMLOG/CONELOG SCREW-LINE surgery set contains new drills without internal cooling, in a design with four cutting edges offering an outstanding cutting performance.

CONELOG SCREW-LINE implants have a self-locking internal taper $\left(7.5^{\circ}\right)$ and the popular CAMLOG indexing with three grooves in the implant and the matching cams on the abutment. This makes for user-friendly handling and a high accuracy of fit for the system parts. In the CONELOG Implant System, the taper is used only with the abutments thus height displacement in impression-taking is excluded. A clever release tool makes loosening the abutment an easy task.

CONELOG SCREW-LINE implants are available with diameters of $3.3 \mathrm{~mm}$, $3.8 \mathrm{~mm}, 4.3 \mathrm{~mm}$, and $5.0 \mathrm{~mm}$. A $7 \mathrm{~mm}$ short implant is available for diameters $3.8 \mathrm{~mm}, 4.3 \mathrm{~mm}$, and $5.0 \mathrm{~mm}$. The implant lengths $9 \mathrm{~mm}, 11 \mathrm{~mm}, 13$ $\mathrm{mm}$, and $16 \mathrm{~mm}$ are available for all implant diameters.

\section{Reader response number 55}

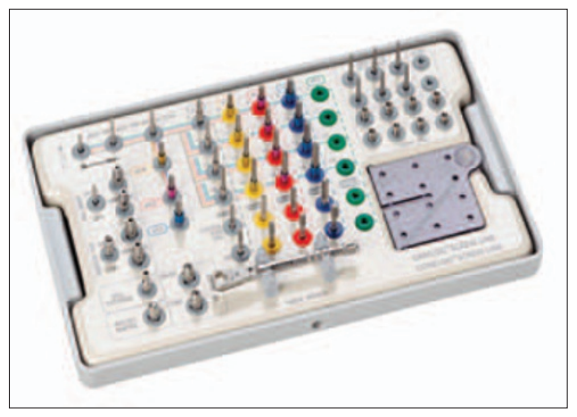

